\title{
Article \\ Gibberellin Oxidase Gene Family in L. chinense: Genome-Wide Identification and Gene Expression Analysis
}

\author{
Lingfeng Hu ${ }^{1}$, Pengkai Wang ${ }^{2}$, Zhaodong Hao ${ }^{1}$, Ye Lu ${ }^{1}$, Guoxia Xue ${ }^{1}$, Zijian Cao ${ }^{1}$, Haoxian Qu ${ }^{1}$, \\ Tielong Cheng ${ }^{1}$, Jisen Shi ${ }^{1} \mathbb{D}$ and Jinhui Chen ${ }^{1, *}$ \\ 1 Key Laboratory of Forest Genetics and Biotechnology of Ministry of Education, Co-Innovation Center for \\ Sustainable Forestry in Southern China, Nanjing Forestry University, Nanjing 210037, China; \\ hlf625@nifu.edu.cn (L.H.); haozd1992@163.com (Z.H.); luye@njfu.edu.cn (Y.L.); xueguoxia@njfu.edu.cn (G.X.); \\ caozijianv@163.com (Z.C.); quhaoxian@163.com (H.Q.); chengt1@njfu.edu.cn (T.C.); jshi@njfu.edu.cn (J.S.) \\ 2 College of Horticulture Technology, Suzhou Polytechnic Institute of Agriculture, Suzhou 215000, China; \\ mengxiao02181@hotmail.com \\ * Correspondence: chenjh@njfu.edu.cn; Tel.: +86-025-85428817-83
}

Citation: Hu, L.; Wang, P.; Hao, Z.; Lu, Y.; Xue, G.; Cao, Z.; Qu, H.;

Cheng, T.; Shi, J.; Chen, J. Gibberellin Oxidase Gene Family in L. chinense: Genome-Wide Identification and Gene Expression Analysis. Int. J. Mol. Sci. 2021, 22, 7167. https://doi.org/ $10.3390 /$ ijms 22137167

Academic Editor: Bartolome Sabater

Received: 13 June 2021

Accepted: 28 June 2021

Published: 2 July 2021

Publisher's Note: MDPI stays neutral with regard to jurisdictional claims in published maps and institutional affiliations.

Copyright: (C) 2021 by the authors. Licensee MDPI, Basel, Switzerland. This article is an open access article distributed under the terms and conditions of the Creative Commons Attribution (CC BY) license (https:// creativecommons.org/licenses/by/ $4.0 /)$.

\begin{abstract}
GAox is a key enzyme for the transformation of gibberellins, and belongs to the 2ketoglutarate dependent dioxygenase gene family (2ODD). However, a systematic analysis of GAox in the angiosperm L. chinense has not yet been reported. Here, we identified all LcGAox gene family members in L. chinense, which were classified into the three subgroups of GA20ox, C19GA2ox, and C20GA2ox. Comparison of the gene structure, conserve motifs, phylogenetic relationships, and syntenic relationships of gibberellin oxidase gene families in different species indicated that the gene functional differences may be due to the partial deletion of their domains during evolution. Furthermore, evidence for purifying selection was detected between orthologous GAox genes in rice, grape, Arabidopsis, and L. chinense. Analysis of the codon usage patterns showed that mutation pressure and natural selection might have induced codon usage bias in angiosperms; however, the LcGAox genes in mosses, lycophytes, and ambarella plants exhibited no obvious codon usage preference. These results suggested that the gibberellin oxidase genes were more primitive. The gene expression pattern was analyzed in different organs subjected to multiple abiotic stresses, including GA, abscisic acid (ABA), and chlormequat (CCC) treatment, by RNA-seq and qRT-PCR, and the stressand phytohormone-responsive cis-elements were counted. The results showed that the synthesis and decomposition of GA were regulated by different $L c G A o x$ genes in the vegetative and reproductive organs of $L$. chinense, and only $L c G A 2 o x 1,4$, and 7 responded to the $\mathrm{NaCl}$, polyethylene glycol, $4{ }^{\circ} \mathrm{C}$, $\mathrm{GA}, \mathrm{ABA}$, and CCC treatment in the roots, stems, and leaves of seedlings at different time periods, revealing the potential role of $L c G A o x$ in stress resistance.
\end{abstract}

Keywords: gibberellin oxidase; L. chinense; gene family; abiotic stress; phytohormone

\section{Introduction}

As a class of tetracyclic diterpenes, gibberellins (GA) are indispensable for the growth and development of plants. Some bioactive GAs are widely involved in various biological activities [1], such as seed germination [2], stem and shoot elongation [3,4], leaf expansion [5], and late flower and fruit development [6,7]. The regulatory synthesis pathway of GA has been studied in depth in model plants, and the related regulatory genes have been successively confirmed by scientists [8]. The GA synthesis pathway can be divided into three main steps: the first step is the catalysis of geranylgeranyl pyrophosphat (GGPP) from ent-kaurene by the enzymes of CPS and KS in the piroplasmid; the second step is the synthesis of GA12 according to $\mathrm{KO}$ and $\mathrm{KAO}$ enzymes by the products of the previous step in the endomembrane; and the third step is the synthesis of physiologically active GA under the catalysis of the gibberellin oxidase (GAox) enzyme [9-11]. 
Liriodendron is an ancient relict with only two species in the genus in nature, including L. chinense and L. tulipifera. Due to their excellent material properties and ornamental value, they are widely planted in China and the United States for landscaping and wood production [12]. The GA signaling pathway plays an important role in promoting plant height and regulating flowering [13]. GAox belongs to the 2OG-Fe (II) oxygenase superfamily, which is divided into multiple small families based on structural and functional differences [11]. The GA20ox, GA2ox, and GA3ox gene families play an important role in GA biosynthesis and degradation [14]. In the last stage of GA biosynthesis, GA 20-oxidase (GA20ox) and GA 3-oxidase ( $G A 30 x)$ are considered to be the key enzymes in the pathways that convert GA12 and GA53 into GA4 and GA1, respectively [6,11]. Increasing or decreasing GA20ox expression levels can improve the fruit setting number and biomass of different species $[7,15,16]$. The loss-of-function of GA20ox in rice was expressed in the shoots but not the reproductive organs, leading to increased grain yields [17]. GA3ox controls plant growth and development mainly by regulating endogenous gibberellin homeostasis [18-22]. GA2ox contains the two subgroups C19GA2ox and C20GA2ox, which are relatively independent in terms of evolution [9]. In tomato, seed germination and development, fruit weight and firmness, and branch growth are all related to GA2ox $[7,23,24]$. Overexpressing GA2ox in Arabidopsis and tobacco could confer dwarfism [25]. The GA 2-oxidase (GA2ox) can preferentially act on physiologically active GA or their inactive precursors to achieve GA degradation [7]. The regulation of GAox expression has also been explored in multiple species. Research has found that GAox plays an important role not only in development and growth but also in the response to stimulation by different exogenous hormones and abiotic stresses [26,27]. OsGA2ox5-OE and AtGA2ox7-OE plants demonstrate tolerance to high-salt treatment [28]. The seedlings of the ga20ox2-1 mutant exhibited primary root and root hair elongation in comparison with the wild-type under $\mathrm{NaCl}$ treatment [26]. Under the influence of GA or GA biosynthesis inhibitors, GAox shows different degrees of functional differentiation and redundancy in cucumbers, grapes, and other species [14,29].

Understanding the GA metabolic regulation pathway in L. chinense can provide a molecular basis for the targeted breeding of new varieties. Gibberellin oxidase is considered to be an important catalyst and synthase responsible for the interconversion of different GAs in the final stage of gibberellin biosynthesis pathway. However, there appear to be no related reports on L. chinense. In this study, we identified 11 GAox gene belonging to the GA20ox and GA2ox bases in the L. chinense genomic database. The LcGAox genes were systematically analyzed in terms of basic physical and chemical properties, gene structure, conserved domains, and selective evolutionary relationships. The evolutionary status of LcGAox was explored via codon usage pattern analysis of GAox genes in 21 species. RNA sequencing (RNA-seq) and quantitative real-time PCR (qRT-PCR) were used to analyze the LcGAox gene expression patterns in multiple organs and under different abiotic stresses, as well as under phytohormone stimulation. This study provides research basis for further understanding the biological function of gibberellin oxidase in L. chinense.

\section{Result}

\subsection{Identification of GA2ox and GA20ox genes in L. chinense}

The GA20ox, GA2ox, and GA3ox genes belong to the 2-oxoglutarate-dependent dioxygenase superfamily in plants and play an important role in the final step of GA biosynthesis. The GAox members of O. sativa, A. thaliana, A. trichopoda, and V. vinifera are listed in Table 1. Through BLASTP and HMMER analysis, 13 candidate genes were obtained from the $L$. chinense genome, including five $L c G A 200 x$ and eight $L c G A 2 o x$ genes. However, GA3ox was not detected in L. chinense. This result indicated that $L c G A 30 x$ was either lost during evolution or was the result of incomplete genome assembly. The LcGA20ox 1-5 and LcGA2ox 1-8 genes were named according to the order of their identification (Table 2). All of the genes were distributed on eight chromosomes except for LcGA2ox2, which was mounted to conting2730. The 1st, 6th, and 9th chromosomes had multiple genes, but only one gene was located on the 2 nd, 3rd, and 9th chromosomes. The coding sequences (CDS) of LcGA20ox 
and LcGA2ox ranged from $732 \mathrm{bp}$ (LcGA2ox6) to $1290 \mathrm{bp}$ (LcGA20ox2). The length of the amino acid sequences ranged from 244 aa to 430 aa. The molecular weight of the LcGAox members ranged from $27.340 \mathrm{kDa}$ to $48.364 \mathrm{kDa}$. The molecular weight differences of the LcGA20ox and LcGA2ox classes were $5.705 \mathrm{kDa}$ and $20.348 \mathrm{kDa}$. The predicted values of the isoelectric points of $L c G A o x$ ranged from 5.31 to 8.60. The results suggest that $L c G A 2 o x 1,2,6$ and $L c G A 200 x 3$ are basic proteins, whereas the remaining proteins are acidic. The predicted subcellular localization results showed that the GA3ox genes in L. chinense were expressed in the cytoplasm.

Table 1. Number of GAox genes in five species.

\begin{tabular}{cccccc}
\hline Species & GA20ox & GA2ox & GA3ox & GAox & Total \\
\hline O. sativa & 5 & 11 & 2 & 3 & 21 \\
A. thaliana & 5 & 7 & 4 & 0 & 16 \\
A. trichopoda & 4 & 5 & 1 & 1 & 11 \\
V. vinifera & 2 & 6 & 3 & 0 & 11 \\
L. chinense & 5 & 8 & 0 & 0 & 13 \\
\hline
\end{tabular}

Table 2. Summary of $L c G A 0 x$ gene family characters.

\begin{tabular}{|c|c|c|c|c|c|c|c|c|c|}
\hline Gene ID & Gene Name & $\begin{array}{l}\text { Accession } \\
\text { Number }\end{array}$ & Position & Location & CDS (bp) & Peptide (aa) & Mw (kDa) & pI & $\begin{array}{r}\text { Subcellular } \\
\text { Localization }\end{array}$ \\
\hline Lchi18432 & LcGA20ox1 & MZ361712 & Chr6 & $41600656-41604092$ & 1245 & 415 & 46.976 & 5.93 & Cytoplasm \\
\hline Lchi25954 & LcGA20ox2 & MZ361709 & Chr6 & $38707502-38709176$ & 1290 & 430 & 48.367 & 5.97 & Cytoplasm \\
\hline Lchi01764 & LcGA20ox3 & MZ361716 & Chr2 & $73946076-73948031$ & 1125 & 375 & 42.662 & 8.36 & Cytoplasm \\
\hline Lchi01394 & LcGA20ox4 & MZ361713 & Chr11 & $56684039-56686083$ & 1149 & 383 & 43.514 & 5.78 & Cytoplasm \\
\hline Lchi10458 & $L c G A 200 \times 5$ & MZ361707 & Chr1 & 79200174-79213961 & 1197 & 399 & 45.202 & 6.1 & Cytoplasm \\
\hline Lchi04083 & LcGA2ox1 & MZ361710 & Chr15 & $63254044-63287148$ & 1014 & 338 & 38.043 & 8.18 & Cytoplasm \\
\hline Lchi35277 & LcGA2ox2 & MZ361718 & Contig2730 & $15814-18566$ & 1005 & 335 & 37.624 & 7.59 & Cytoplasm \\
\hline Lchi07023 & LcGA2ox3 & MZ361714 & Chr3 & $21930812-21932403$ & 996 & 332 & 37.241 & 6.41 & Cytoplasm \\
\hline Lchi12922 & $L c G A 20 \times 4$ & MZ361717 & Chr1 & $8453778-8461759$ & 771 & 257 & 29.734 & 6.34 & Cytoplasm \\
\hline Lchi29789 & LcGA2ox5 & MZ361719 & Chr4 & $67227159-97231797$ & 1011 & 337 & 38.25 & 5.83 & Cytoplasm \\
\hline Lchi00133 & LcGA2ox6 & MZ361715 & Chr9 & $2749738-2755769$ & 732 & 244 & 27.34 & 8.6 & Cytoplasm \\
\hline Lchi15175 & LcGA2ox7 & MZ361708 & Chr9 & $52008101-52009229$ & 978 & 326 & 36.906 & 5.76 & Cytoplasm \\
\hline Lchi18410 & LcGA2ox 8 & MZ361711 & Chr6 & $42633756-42642514$ & 1272 & 424 & 47.688 & 5.31 & Cytoplasm \\
\hline
\end{tabular}

\subsection{Evolutionary Analysis of LcGA20ox and LcGA2ox Genes}

A phylogenetic tree was constructed based on the full-length protein sequences of GA3ox in L. chinense (Lc), O. sativa (Os), A. thaliana (At), A. trichopoda (Atr), and V. vinifera (Vv) (Figure 1). According to previous research [30], the sequences were divided into five subgroups, namely GA20ox, GA3ox, GAox, C19GA2ox, and C20GA2ox. As there were no GA3ox genes found in the GA3ox and GAox subgroups of L. chinense, we will only refer to the phylogenetic relationships of GA20ox, C19GA2ox, and C20GA2ox in the five species. The results showed that GA20ox, C19GA2ox, and C20GA2ox in each species were distributed in different subfamilies (Figure 1). LcGA20ox included five members, namely LcGA2ox1,2,3,6, which belonged to C19GA2ox, and LcGA2ox4,5,7,8 was classed into the C20GA2ox subfamily. The phylogenetic tree result indicated that most members of $L c G A 20 x$ and $L c G A 200 x$ were clustered into a clade with $V v G A 200 x, V v G A 20 x, A \operatorname{trGA20ox}$, and $A \operatorname{trGA2ox}$. This indicated that the GA3ox of L. chinense, $V$. vinifera, and $A$. trichopoda could have originated from the same ancestor, except for LcGA2ox5. In the C19GA2ox subgroup, $L c G A 20 x 1,2,3$ shared high similarities, indicating that they could have functional consistency, and the other members in the different subfamilies may have formed functional specializations during evolution. 


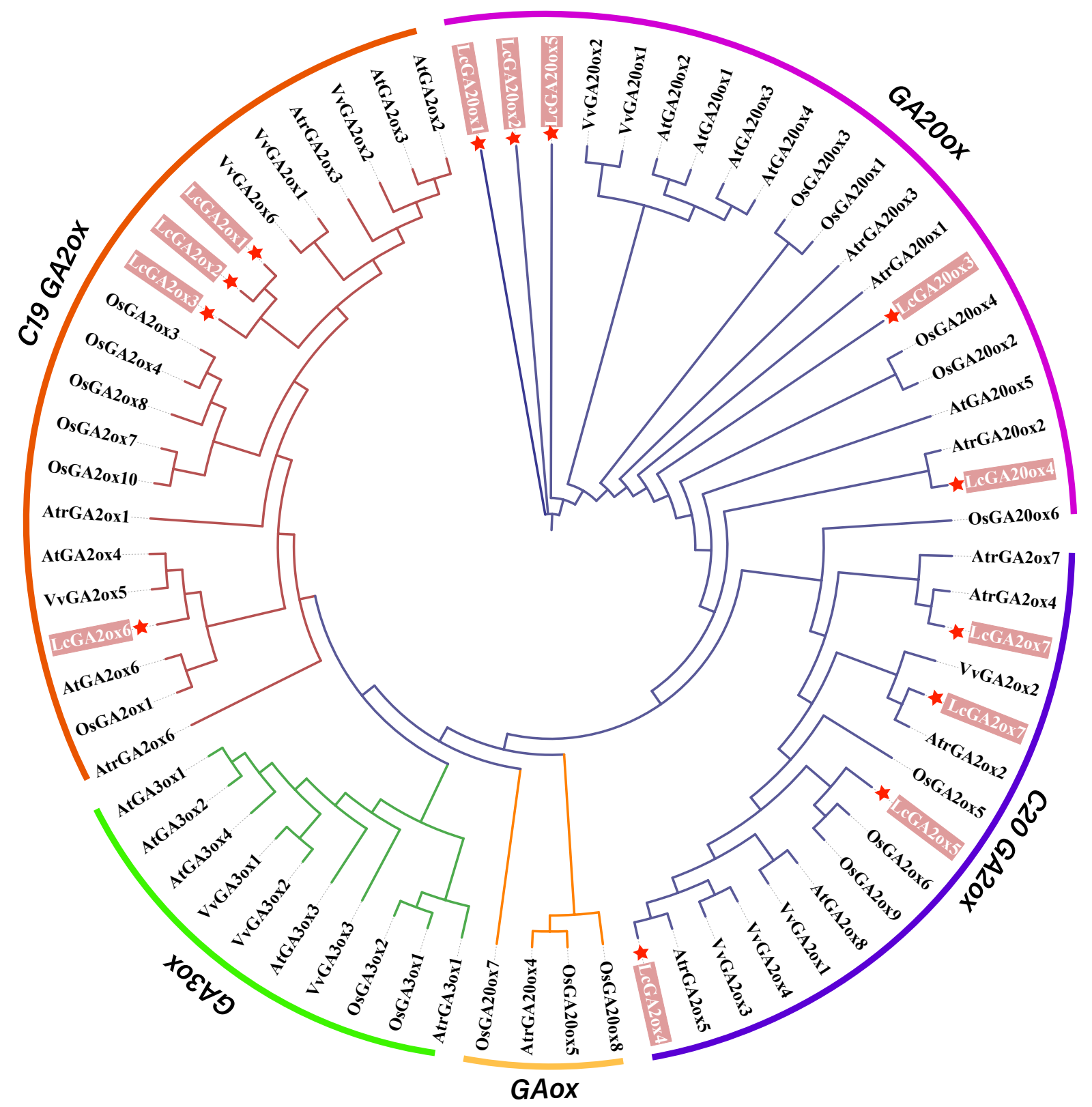

Figure 1. Phylogenetic analysis of gibberellin oxidase in L. chinense (Lc), O. sativa (Os), A. thaliana (At), A. trichopoda (Atr), and $V$. vinifera $(\mathrm{Vv})$. The amino acid sequences of GA20ox, GA2ox, and GA3ox were aligned with ClustalX, and phylogenetic tree was constructed using maximum likelihood (ML) method in IQtree2.0.5 with 1000 bootstrap replicates, the LG + F + R6 was selected to the optimal model. The gibberellin oxidase genes in L. chinense were showed by star in red.

The evolution of gene function is related to changes in gene structure. The GAox protein conserved motif and gene structure of L. chinense and A. thaliana were analyzed (Figures 2 and S1). The GAox family protein contained two conserved domains: 2OG-FeII oxygenase and DIOX_N, which play important roles in the synthesis of GA in plants [14]. To investigate the motifs shared among related proteins within the same subfamily, the 15 distinct motifs were predicted by MEME software (Figures 2 and S1). In the prediction result, motifs $1,2,3,5,6,8,9,11$, and 12 were identified in most of the GAox proteins, except the individual LcGAox. Motifs 4, 13, and 14 only existed in GA20ox, and motif 7 was predicted in GA20ox and GA3ox. Motifs 10 and 15 were unique to C19GA2ox and C20GA20x, respectively. Interestingly, motif 9 was located in the N-terminal of GA20ox and in the C-terminal of the other GAox proteins. Motifs 1, 7, and 12 belong to the DIOX_N domain, and motifs 2, 3, 5, 6, and 11 belong to the 2OG_FeII oxygenase domain. We found 
that some specific motifs were absent or increased in L. chinense. Motif 12 was absent in LcGA20x8. Motifs 2 and 5 were absent in LcGA2ox4, and motif 11 was absent in LcGA2ox6. In addition to the motifs of the two domains, the specific motif 10 in C19GA20x was identified in LcGA20ox4. The GA20 oxidase contained a conserved GA substrate-binding motif and 2-oxoglutarate-binding motif. According to previous research, the amino acid residues that bind the $\mathrm{Fe}^{2+}$ and interact with the 5-carboxylate of 2-oxoglutarate belong to GA oxidase [31,32]. The multiple sequence alignments and 3D structure results showed that the GA substrate-binding motif (NYYPPXCXXP) and 2-oxoglutarate-binding motif (LPWKET) are also ubiquitous in LcGA20ox genes (Figure 3). The amino acid residues with specific functions were conserved in LcGAox and AtGAox, except for LcGA2ox4 and 6, in which the HXD dyad near the amino terminus is lost (Figures 3 and S2). Summarizing the above results, the $L c G A o x$ and $A t G A o x$ superfamily possesses common conserved domains and sites. However, the amino acid sequences of the domains are diverse, indicating that functional differentiation may be present between the two.

The gene structure analysis results of GAox in L. chinense and A. thaliana are indicated in Figure 3. The number of exons in most GAox genes was between 2 and 3. AtGA3ox2 only had one exon, while LcGA2ox5 and LcGA20ox1 had four exons. Most GAox genes had similar gene structures, but were found to possess functional conservatism. Interestingly, LcGA20x1 had a very long intron near the C-terminal (about $30 \mathrm{~kb}$ ), which may be caused by genome annotation issues or the presence of transposable elements; an observation that needs to be further verified.
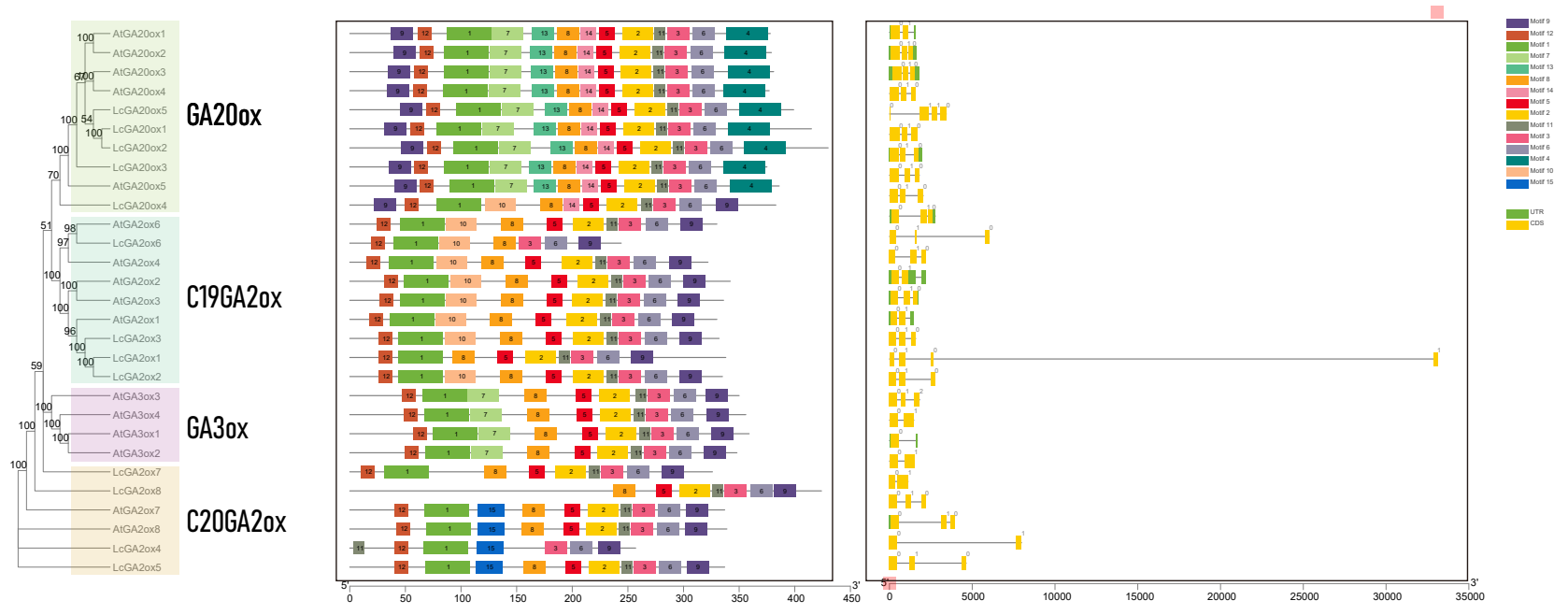

Figure 2. Evolutionary relationships, conserved protein motifs and gene structure in GAox genes from L. chinense and A. thaliana. The phylogenetic tree was constructed using the neighbor joining (NJ) method with 1000 bootstrap replicates in MEGA 7.0. All protein sequences of gibberellin oxidase in L. chinense and A. thaliana were divided to four subgroups. Different colors represent different subgroup. The numbers 1-15 of motif composition was displayed in different colored boxes in LcGAox and AtGAox proteins. Gene structure was showed off in the right box, the yellow box represents the coding sequences and the green box represent the UTR sequences. 

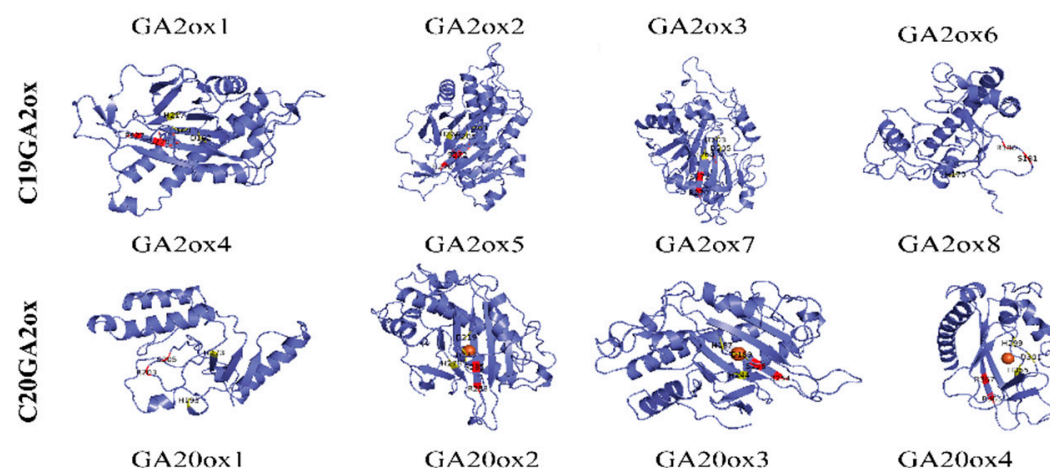

GA20x 8

GA200x2

GA200x3

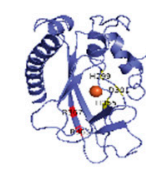

GA $200 \times 4$

GA200x 5
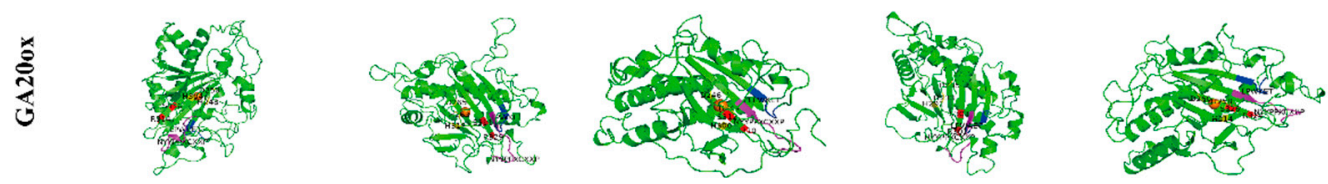

Figure 3. 3D structures of LcGA oxidases showing functional sites. The conserved motif related GA substrate binding site and 2-oxoglutarate-binding motif were display the purple and blue bands. The amino acid residues that bind the $\mathrm{Fe}^{2+}$ and interacted with the 5-carboxylate of 2-oxoglutarate are highlighted in yellow and red, respectively.

\subsection{Synteny Analysis of GAox Genes in L. chinense, Grape, Arabidopsis, and Rice}

Whole-genome duplications, segmental duplications, and tandem duplications play important roles in gene family expansion. Thus, we analyzed the duplication events of GAox in L. chinense. In the L. chinense genome, two pairs of segmentally duplicated genes were identified: $L c G A 20 \times 3 / L c G A 20 \times 1$ and $L c G A 20 \times 3 / L c G A 20 \times 6$ (Figure 4a). This result indicates that the $L c G A 20 x$ gene family was amplified via the segmental duplication of genes, whereas no duplication events were found in LcGA20ox. To further explore the evolutionary relationships of the GAox gene family, we performed collinearity analysis among L. chinense and three other species (Arabidopsis, grape, and rice). The results indicated that most of the orthologous GAox genes were distributed into multiple chromosomes, except in rice, which had only one location for Os-1 (Figure $4 b$ ). In Lc/At, Lc/Os, and Lc/Vv, the number of orthologous genes in $\mathrm{Lc} / \mathrm{Vv}$ was much higher than in the other two groups, indicating that the relationship between grape and Liriodendron was closer in the GAox gene family.

a

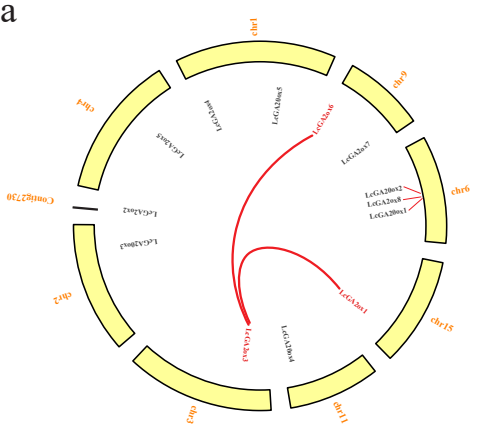

b

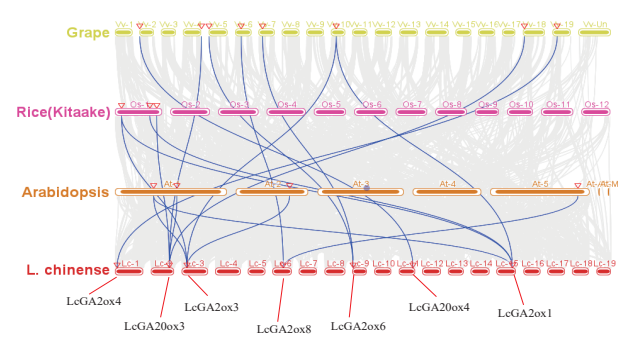

Figure 4. Genome-wide synteny analysis of GA2ox and GA2ox gene family among L. chinense and other three species. (a): Distribution and segmental duplication of GAox genes in L. chinense. The yellow panel shows the 8 chromosomes using a circle, and the contig are display through the black line. Red lines connecting homologous genes; chromosome numbers are marked outside of the circle. (b): Synteny analysis of GAox genes between L. chinense, Arabidopsis, grape, and rice (Kitaake). Gray lines in the background indicate the collinear blocks within L. chinense, Arabidopsis, grape, and rice (Kitaake) genomes, while the blue lines highlight the syntenic GAox gene pairs. Different gene pairs were highlighted by red triangle. 
To analyze the evolutionary selection in the LcGAox gene family, we counted the nonsynonymous (Ka) and synonymous substitutions (Ks) among the orthologous gene pairs, as well as the $\mathrm{Ka} / \mathrm{Ks}$ ratios among the four species (Table 3). The results showed that the genes of the duplicate pairs had KA/Ks ratio $<1$, with no ratio $>1$, and the KA/KS ratio within species was less than the ratio between species. This suggested that the orthologous GAox genes of the four species were subjected to purifying selection during evolution.

Table 3. The orthologous gene pairs' $\mathrm{Ka} / \mathrm{Ks}$ ratios among four species.

\begin{tabular}{|c|c|c|c|c|c|}
\hline \multicolumn{3}{|c|}{ Duplicated GAox Gene Pairs } & \multirow{2}{*}{$\begin{array}{c}\text { Ка } \\
0.1752\end{array}$} & \multirow{2}{*}{$\begin{array}{c}\text { Ks } \\
0.7673\end{array}$} & \multirow{2}{*}{$\begin{array}{c}\mathrm{Ka} / \mathrm{Ks} \\
0.2283\end{array}$} \\
\hline & LcGA2ox1 & $L c G A 2 o x 3$ & & & \\
\hline $\mathrm{Lc} / \mathrm{Lc}$ & $\operatorname{LcGA2ox3}$ & $\operatorname{LcGA2ox6}$ & 0.5347 & 1.5333 & 0.3487 \\
\hline \multirow{3}{*}{$\mathrm{Lc} / \mathrm{At}$} & LcGA2ox1 & AT1G30040 (AtGA2ox2) & 0.7197 & 0.9484 & 0.7589 \\
\hline & LcGA20ox3 & AT1G44090 (AtGA20ox5) & 0.5312 & 1.0585 & 0.5018 \\
\hline & LcGA2ox8 & AT5G58660 & 0.6419 & 0.8638 & 0.7431 \\
\hline \multirow{8}{*}{$\mathrm{Lc} / \mathrm{Vv}$} & LcGA2ox4 & VIT_219s0177g00020 & 1.4737 & 1.5654 & 0.9414 \\
\hline & LcGA20ox4 & VIT_202s0234g00010 & 0.2196 & 0.686 & 0.3201 \\
\hline & LcGA2ox1 & VIT_210s0003g03490 & 0.5232 & 0.6875 & 0.761 \\
\hline & LcGA20ox3 & VIT_218s0001g01390 & 0.2933 & 0.525 & 0.5587 \\
\hline & LcGA20ox3 & VIT_204s0044g01650 & 0.3052 & 0.7457 & 0.4093 \\
\hline & LcGA2ox8 & VIT_206s0004g06790 & 0.5502 & 0.8525 & 0.6454 \\
\hline & LcGA2ox6 & VIT_205s0077g00520 & 0.5477 & 1.3039 & 0.42 \\
\hline & LcGA2ox6 & VIT_207s0005g01920 & 0.5626 & 1.0286 & 0.547 \\
\hline \multirow{3}{*}{$\mathrm{Lc} / \mathrm{Os}$} & LcGA2ox1 & OsKitaake01g330800 & 0.775 & 0.9395 & 0.8249 \\
\hline & LcGA2ox1 & OsKitaake01g077300 & 0.77 & 1.2028 & 0.6402 \\
\hline & LcGA20ox3 & OsKitaake01g424900 & 0.351 & 0.9514 & 0.3689 \\
\hline
\end{tabular}

\subsection{Codon Usage Pattern Analysis in GAox Genes}

The codon usage pattern reflects the evolution and mutation of species or genes and also plays an important role in gene expression level and functional differentiation [33,34]. By analyzing the GA20ox, C19GA20x, and C20GA2ox subgroup genes in 21 species (approximately 391 sequences; Table S2), we constructed relatively systematic codon usage patterns in the gibberellin oxidase gene family. Codon usage bias (CUB) is largely affected by the whole base content of each gene, and the overall GC content often reflects the strength of directional mutation, and the main difference of the synonymous codon is typically reflected in the third base [35]. The basic statistics of the third base are indicated in Figure 5. The usage percentage of G/C in the third base was obviously less than A/T in the dicotyledons, and the G3s/C3s ratio was much higher than the A3s/T3s ratio in the monocotyledons. Among the magnoliaceous plants represented by L. chinense and the Amborella, the usage percentage of G3s/C3s and A3s/T3s was almost equal. The third base usage model among mosses and lycophytes was similar to the monocots. This suggested an unequal distribution of $\mathrm{A}, \mathrm{T}, \mathrm{C}$, and $\mathrm{G}$, as well as different biases during plant evolution. We estimated Karl Pearson's correlation coefficients between the nucleotide composition at the third codon position and GC12 to analyze the codon usage pattern in the GAox gene family (Figure 5). In all 21 species, significant positive correlations were detected between C3s, G3s, GC, GC3s, and GC12s, indicating that the codon usage pattern was selected by mutation pressure in the GAox gene family. The ENC is an effective indicator for evaluating the overall codon preference of codon genes, and we used ENC as the ordinate and GC3s as the abscissa to construct an ENC plot to explore the relationship between base composition and codon preference (Figure 6a). The result showed that the ENC values of GA20ox and GA2ox ranged from 40 to 60, except for in the monocotyledons. Most of the GAox genes in the mosses, lycophytes, Amborellaceae, and L. chinense (Magnoliaceae) had ENC values that were basically around 50-60, showing no obvious codon bias (Figure 6a). However, for GA20ox and GA2ox in the monocots, the gene ENC values were generally distributed around 35, except for some C19GA2ox genes, indicating that the gene codons had significant usage preference. ENC plots are widely used to analyze the main factors affecting 
codon usage [28]. Most of the points were in close proximity to the curve (Figure S3), indicating that mutation pressure played an important role in the codon usage bias, though natural selection and other factors could have had a certain influence. Parity rule 2 (PR2) is considered to be an important index that assesses whether base bias in different mutation and selection pressures exists in the nucleotide composition. In the GA20ox and C19GA2ox gene subgroups, the third position of the codon $\mathrm{T}$ was used more frequently than $\mathrm{A}$, and $\mathrm{C}$ was used more frequently than $\mathrm{G}$ (Figure $6 \mathrm{~b}$ ). The frequency of nucleotide A of $L c G A 200 x$ was higher than that of $\mathrm{T}$, but this result was in contrast to that of the LcC19GA2ox genes. The trend of base usage frequency of L. chinense was consistent with the overall C20GA2ox gene subfamily and was in the order of T > A, G > C. In summary, A and T were not equal to $\mathrm{G}$ and $\mathrm{C}$, and a few genes were close to the center, suggesting that natural selection and mutation pressure might have influenced the CUB of the GA20ox, C19GA20x, and C20GA2ox gene subfamily.

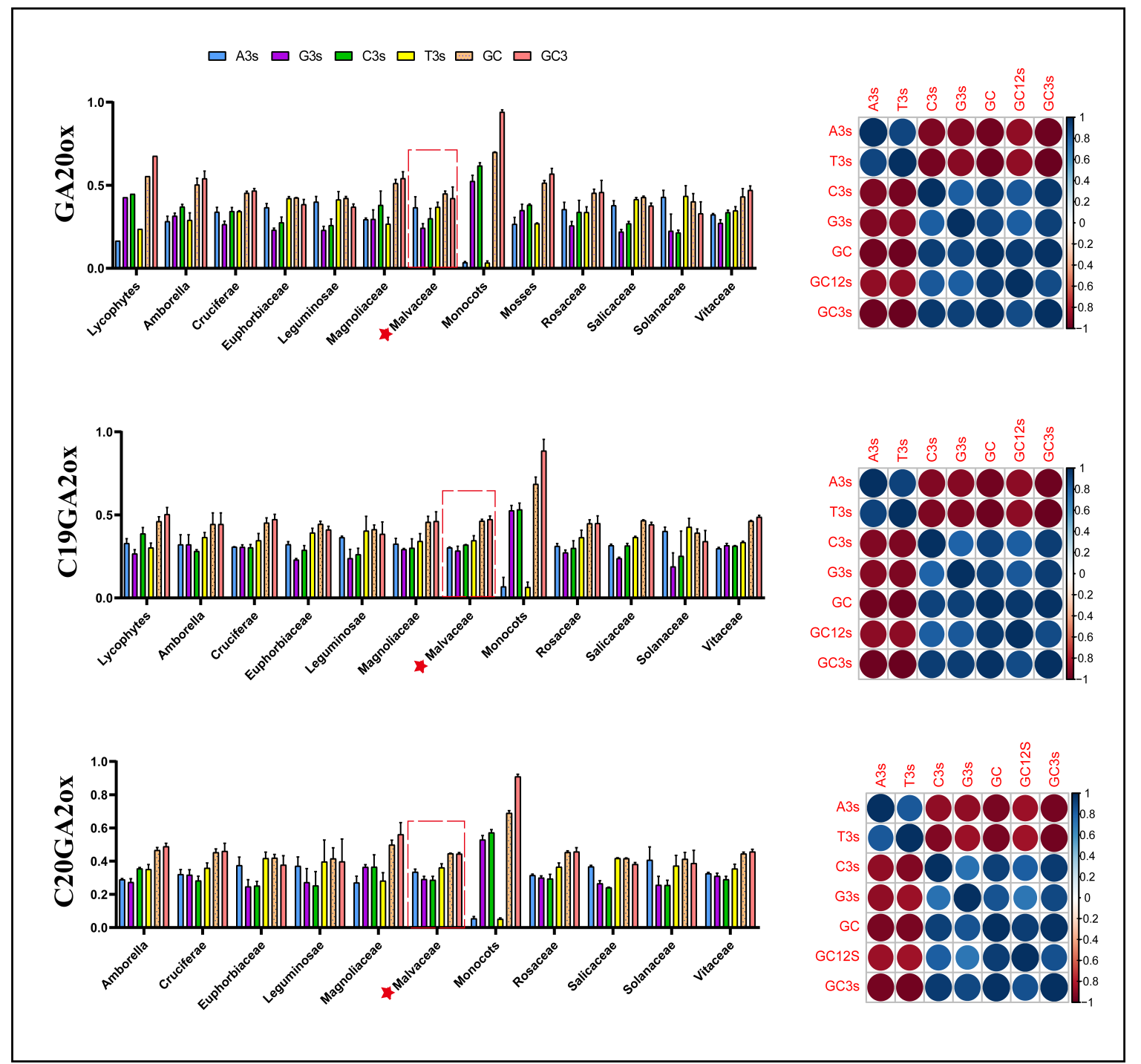

Figure 5. The bases in the third statistics and correlation analysis of GA20ox, C19GA2ox, and C20GA2ox in 21 species. The different color represents the third base frequency in codon. The red star and red dotted frame represent the L. chinense frequency of related bases in GA20ox, C19GA2ox, and C20GA2ox. 


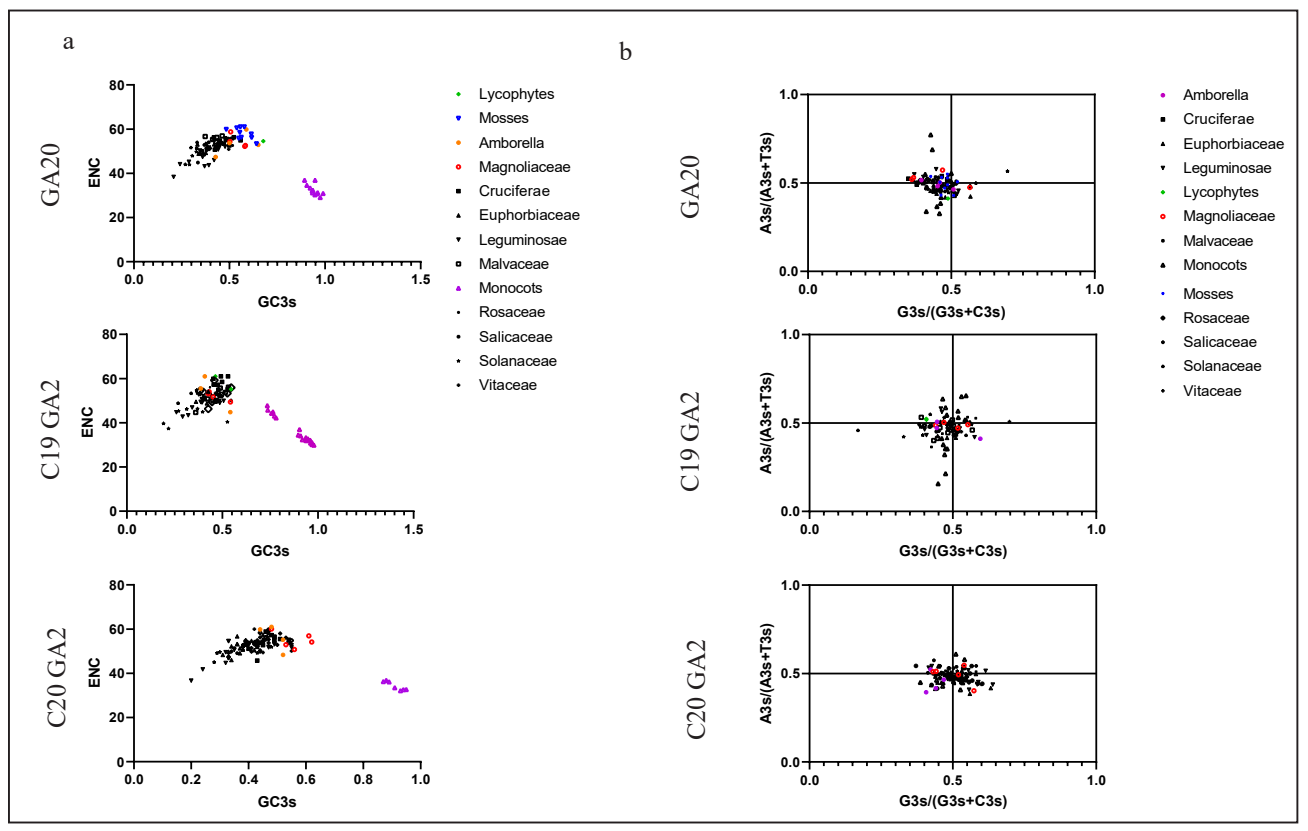

Figure 6. ENC-plot analysis and PR2-plot analysis in GA20ox, C19GA2ox, and C20GA2ox. (a): The points represent a single gene, ENC value of each gene as the ordinate and the GC3s value as the abscissa. Different shapes represent different families, and blue inverted triangle represents mosses, the green diamond represents lycophytes, the organ filled circle represents Amborella, the purple triangles represent monocots and the hollow red circle represents L. chinense. (b): This plot is tested with AT bias (3s/A3s + T3s) in the y-axis and GC bias (G3s/(G3s + C3s) in the x-axis in a graphical presentation. Different color and shapes represent different families.

We performed RSCU analysis of codons to better understand the patterns of codon usage in the GAox gene family. Among the 59 synonymous codons in the GA20ox and GA2ox gene families of all 21 species (Table S5), we found that some codons exhibited no obvious preference (RSCU $=1$ ), but in different subgroups, some special codons were found in different species. For example, AAG, CAC, GAT, and GAA had significant preferences (RSCU > 2) in Cruciferae. In the GA2ox subgroups, AGG and AGA (RSCU > 2), exhibited usage bias in most species, except for monocots (Figure 7).

The above analysis results indicated that the GA20ox, C19GA2ox, and C20GA2ox gene subgroups exhibited comparatively more bias towards A/T-ending codons compared to G/C-ending codons in most species, but the monocotyledon nucleotide composition biases of the third were conversed with the other species, with strong codon preference. This base composition pattern, which differs significantly from dicotyledonous plants, may be due to mutation pressure and natural selection. Liriodendron chinense had similar base composition patterns as A. trichopoda, as well as no obvious preference for codon usage an aspect that differs between monocotyledonous and dicotyledonous plants, thus indicating that the LcGAox genes may be more primitive. 

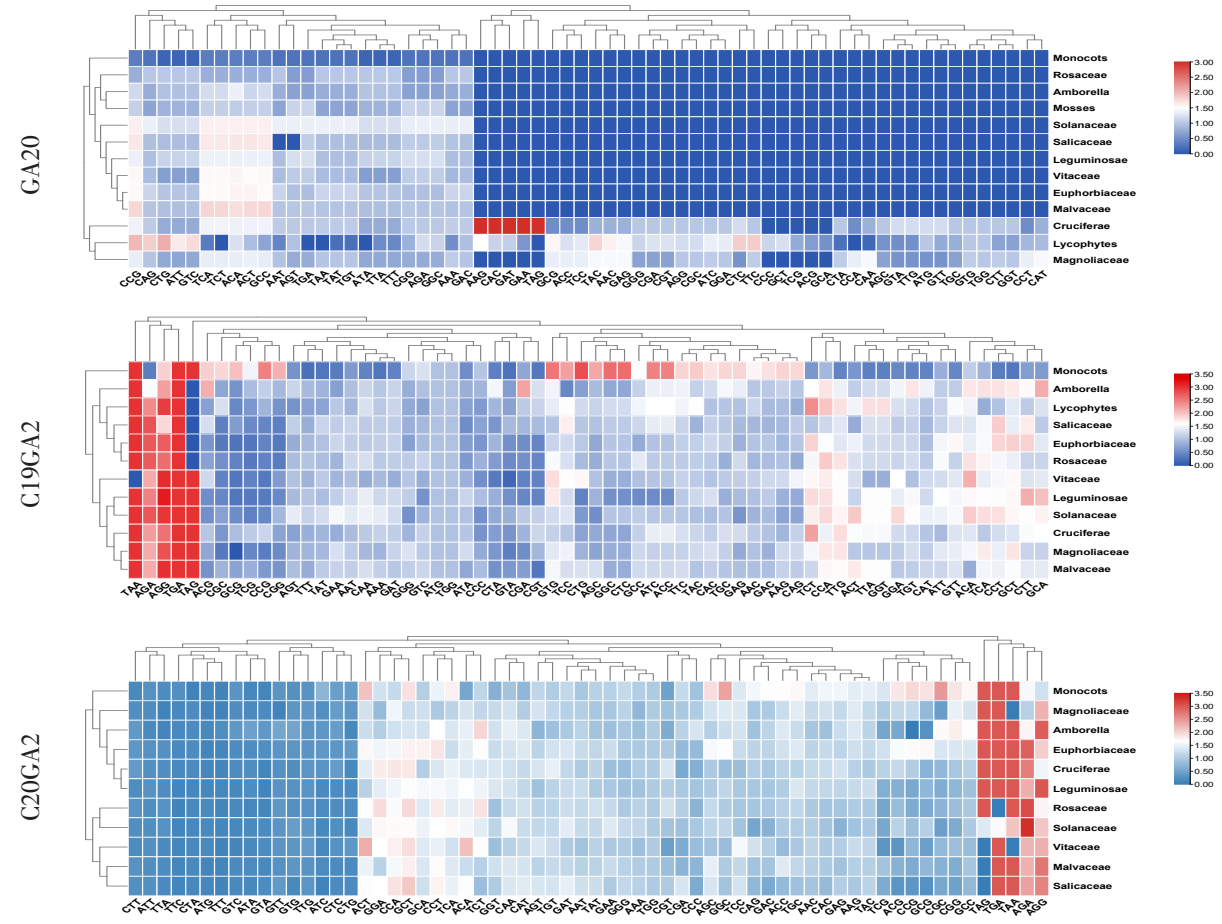

Figure 7. Relative synonymous codon usage (RSCU) of GA20ox, C19GA2ox, and C20GA2ox gene families in all 21 species. Blue-to-red color indicates low to high RSCU values of codons.

\subsection{Cis-Elements in the Promoters of LcGA2ox and LcGA20ox}

The cis-regulatory element in the promoter region determines the binding specificity of the transcription factor, and, thus, it has the role of transcription regulation. PlantCARE was used to predict the cis-elements related to stress responsiveness and phytohormone responsiveness to analyze the functions of $L c G A 200 x$ and LcGA2ox (Figure 8). We found that the G-Box and ABRE motifs were ubiquitous and relatively abundant in all the gibberellin oxidases of $L$. chinense, and in comparison, with other $L c G A o x$ genes, the number was higher in the promoters of LcGA20ox2, LcGA20ox5, LcGA2ox1-4, and LcGA2ox7 (Figure 8a). This indicated that the ABRE and G-Box motifs have major functions in the response to stressful environments. These cis-elements were mainly involved in ABA, GA, SA, and auxin responsiveness, as well as light, low temperature, and other stresses (Figure $8 \mathrm{~b}$ ). In general, light-responsive elements are widely present in the $L c G A o x$ gene family, whereas abiotic stress elements are comparatively fewer and only exist in $L c G A 20 x 2,4,5,8, L c G A 20 o x 2,4$, and 5. Different hormones also played a regulatory role in the $L c G A o x$ genes. $L c G A 20 x 1,2,4,5,8$, $L c G A 200 \times 2,4$, and 5 were regulated by SA, and eight genes, including $L c G A 20 \times 2,3,4,5,7$, $L c G A 20 x 1,2$, and 3, were related to auxin. Most genes, except for $L c G A 20 x 8$, were associated with ABA. Although all genes are related to gibberellin oxidase, some genes did not contain gibberellin responsiveness elements in their promoters, including $L c G A 20 x 5$ and LcGA20ox3. 

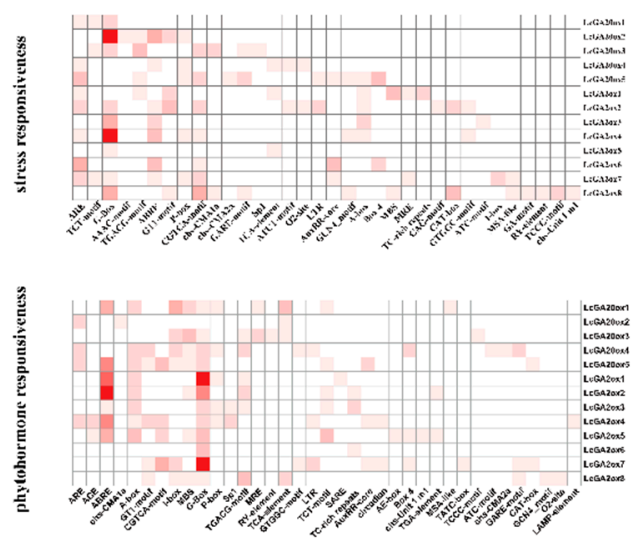

b

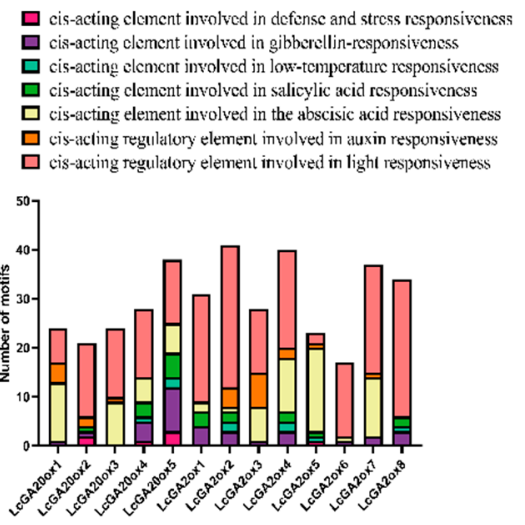

Figure 8. The number of cis-elements in $L c G A 20 o x$ and $L c G A 20 x$ promoter. (a): The combine motifs about phyhormone and stress responsiveness in $L c G A 20 o x$ and $L c G A 20 x$ gene families. The shade of the red represents the quantity. (b): The number of cis-elements about different hormone and abiotic stress in $L c G A 20 o x$ and $L c G A 2 o x$ gene families. Purple represents the gibberellin response element; Pink represents the light response element; Organ represents the auxin response element; Creamy yellow represents the abscisic acid response element; Green represents the salicylic acid response element; Light cyan represents the low-temperature response element; Purple represents the defense and stress response element.

\subsection{Analysis of the Expression Patterns of LcGA2ox and LcGA20ox Genes in Different Organs}

The transcriptome sequencing data of L. chinense were downloaded from the NCBI SRA database, and the gene expression level was obtained using salmon analyses. To elucidate the expression patterns of the LcGA20ox and LcGA2ox genes in L. chinense growth and development, we constructed a gene expression profile in different stages of hybrid Liriodendron somatic embryogenesis and different organs of L. chinense (Figure 9). LcGA2ox1,2,8 and LcGA20ox3 were involved in the process from embryogenic callus to seedling morphogenesis, and $L c G A 20 x 8$ exhibited a trend of high expression levels. During the transition from globular embryo to cotyledon embryo, the expression level of $L c G A 200 x 3$ was downregulated (Figure 9a). LcGA20ox1,4,5, LcGA2ox3,5, and 6 exhibited no or very low expression in the nine organs (Figure 9c). High expression levels of LcGA2ox2 were detected in LP7 (unexpanded leaf) (Figure 9). The expression levels of $L c G A 2 o x 7$ and $L c G A 200 x 2$ were obviously upregulated in the pistils and stamens in comparison with the other organs. LcGA20ox1 was only detected in LP2 and LP7, while LcGA20ox3 and LcGA2ox4 and 8 possessed higher expression levels in the mature leaves, indicating that the different types of gibberellin oxidases participated in the development of the leaves. In the bracts, sepals, petals, and stamens, $L c G A 200 x 3$ and $L c G A 20 x 4$ and 8 were expressed to varying degrees to regulate the balance of internal gibberellin. $L c G A 200 x 3$ and $L c G A 2 o x 4$ and 8 were also detected at the different developmental stages of the petals. The LcGA2ox8 and LcGA20ox3 expression levels remained stable, while the expression level of $L c G A 20 x 4$ was inconsistent with $L c G A 20 x 8$, exhibiting low expression after the S1 stage (Figure $9 \mathrm{~b}$ ). These results showed that the synthesis and decomposition of gibberellin were regulated by different gibberellin oxidases in the vegetative and reproductive organs of L. chinense. 


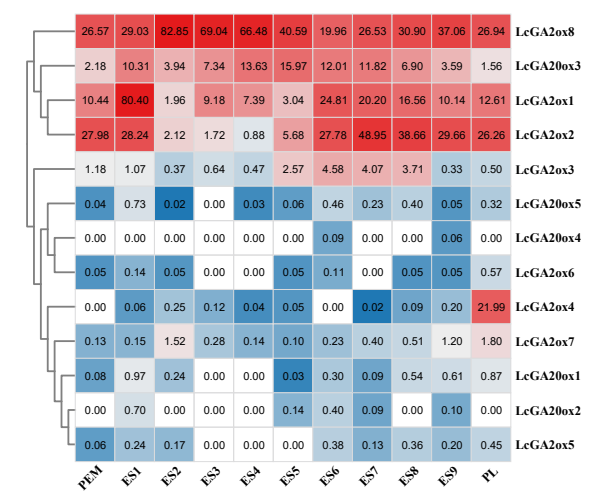

c

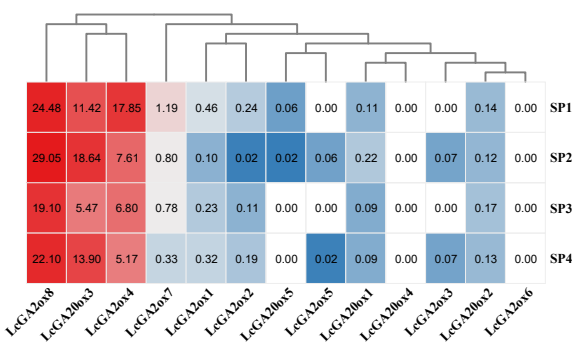

b
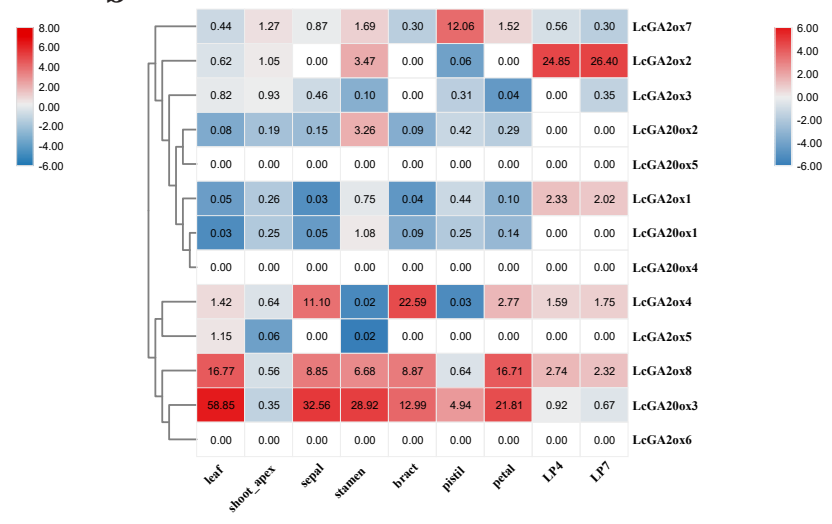

Figure 9. The LcGA20ox and LcGA2ox gene expression profiles in different organs. Two or three biological repeats were shown in the heatmap except bract. The transcripts per million (TPM) was used to indicated the gene expression level. PEM: embryogenic callus; ES1: after 10 days of liquid culture; ES2: two days after sieving; ES3: one day after ABA treatment; ES4: three days after ABA treatment; ES5: globular embryo; ES6: heart shaped embryo; ES7: torpedo embryo; ES8: immature cotyledon embryo; ES9: mature cotyledon embryo; PL: planta; SP1-4: the stage of petals development. (a) and (c) respectively represent the $L c G A o x$ gene expression level during somatic embryogenesis of hybridization liriodendron and the development stage of petal. These RNNA-seq data belong to the lab not publish the data. (b) represents the LcGAox gene expression level in different organs, and the accession numbers has listed in the material and methods 4.5. The expression levels of the genes were expressed by Transcripts Per Million (TPM).

\subsection{Effects of Phytohormone and Abiotic Stress Influence the Expression of LcGA2ox and LcGA20ox}

To investigate whether the LcGA20ox and LcGA2ox gene families participate in the abiotic stress and phytohormone response, the gene expression levels in the different organs of three-month-old seedling were measured under $\mathrm{NaCl}, \mathrm{PEG}, 4{ }^{\circ} \mathrm{C}, \mathrm{GA}, \mathrm{ABA}$, and CCC (chlormequat chloride) treatments by qRT-PCR. The results showed that only LcGA2ox1, LcGA2ox4, and LcGA2ox7 were involved in the above stresses and exhibited a similar expression pattern under most of the treatments (Figure 10).

Under abiotic stress, the expression levels of $L c G A 2 o x 1$ showed an upward trend at first followed by a downward trend under the same treatment (Figure 10 and Table S3). The expression levels of $L c G A 2 o x 1$ and $L c G A 2 o x 4$ reached their peaks at 6-24 $\mathrm{h}$ in the roots, stems, and leaves under PEG and low temperature stress. Under $\mathrm{NaCl}$ stress, the LcGA2ox1 expression level in the roots and leaves peaked at $12 \mathrm{~h}$. Compared with untreated plants, LcGA2ox1 responded to different abiotic stresses far more than normal level (Log2FD > 3) (Table S3). The LcGA2ox4 expression level in the leaves was downregulated compared with $L c G A 2 x o 1$ and was hardly detectable in the roots and stems. After $24 \mathrm{~h}$ of $\mathrm{NaCl}$ stress treatment, LcGA2ox4 expression showed a certain degree of response (Figure 10 and Table S3). It was worth noting that under low temperature stress, LcAG2ox4 had a relatively specific response in leaves, and it has always maintained a relatively stable state. Although $L c G A 2 o x 7$ had different expression patterns under different stresses, the gene relative expression levels changed little overall.

Under the GA and ABA treatments (Figure 10 and Table S3), the expression level of LcGA2ox1 and LcGA2ox4 in the leaves peaked at $24 \mathrm{~h}$ or $12 \mathrm{~h}$, respectively. LcGA2ox7 was 
detected after $48 \mathrm{~h}$ and $6 \mathrm{~h}$ of ABA and GA treatment in the leaves. Almost no expression of $L c G A 20 x 1 / 4$ can be detected in the stem. However, the $L c G A 20 x 7$ expression pattern in the stems was interesting, as within $12 \mathrm{~h}$ after ABA and GA treatment, the expression level was downregulated compared with at $0 \mathrm{~h}$ in the stems. However, the gene expression level suddenly increased at $24 \mathrm{~h}$, following which it began to decline.

After CCC treatment (Figure 10 and Table S3), LcGA2ox4 and LcGA2ox7 expression was suppressed in the stems, but the expression level of $L c G A 20 \times 1$ was upregulated in the stems, with the expression reaching the highest level at $6 \mathrm{~h}$, after which it was downregulated. It was indicated that the active gibberellin or precursor substance degraded by LcGA2ox1 may be different from LcGA2ox4/7. In the leaves, the response of LcGA2ox1 and LcGA2ox4 to CCC was relatively great, peaking at $12 \mathrm{~h}$, following which the expression level decreased.

The above results indicated that in different organ parts of hybrid Liriodendron, the LcGA2ox1/4/7 can respond to abiotic stress and hormone treatment to varying degrees, thereby regulating the balance of gibberellin in the plant to maintain the normal growth.

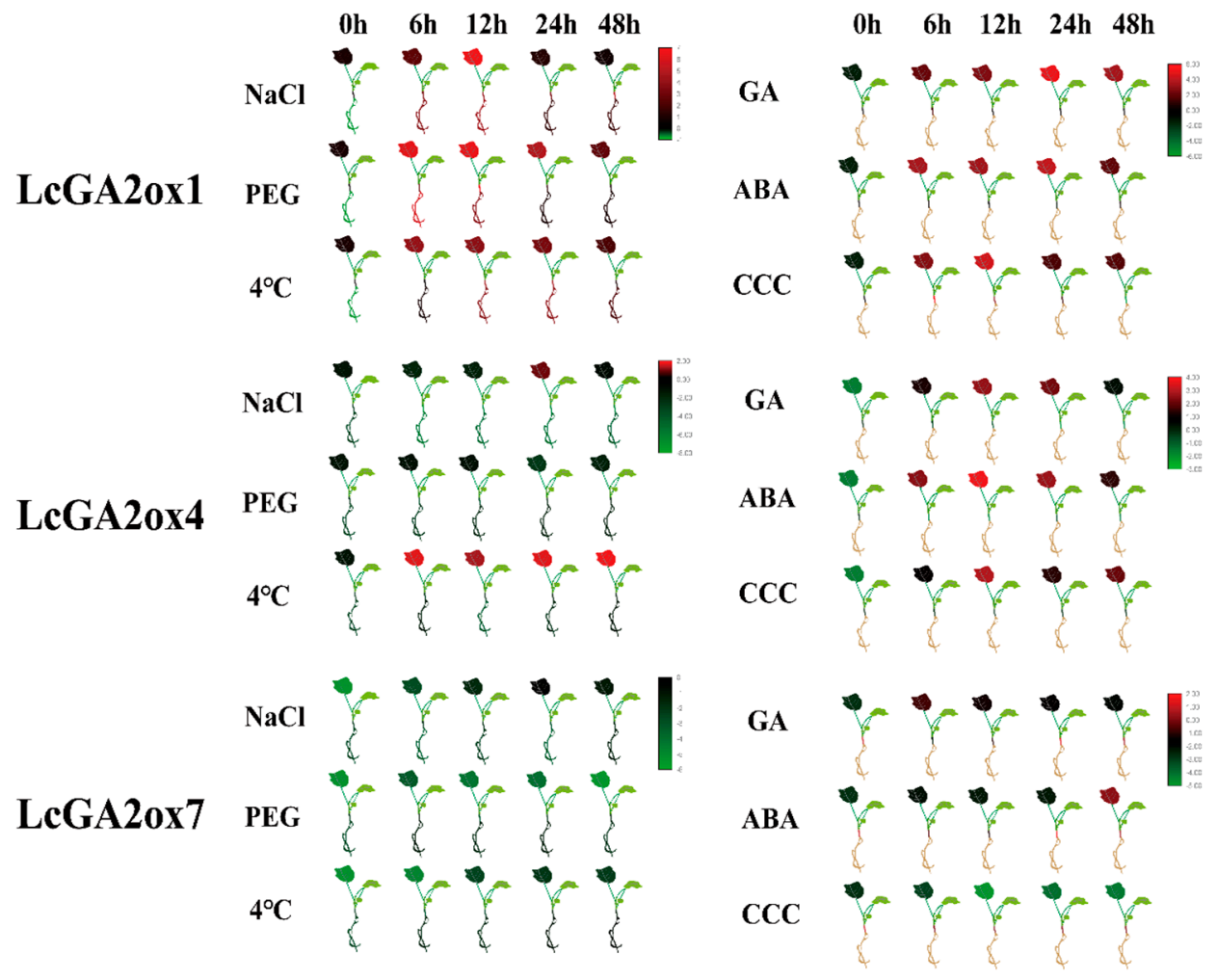

Figure 10. The gene expression level of $L c G A 2 o x 1,4$, and 7 in leaves, stems, and roots under $\mathrm{NaCl}, \mathrm{PEG}, 4{ }^{\circ} \mathrm{C}, \mathrm{ABA}, \mathrm{GA}$, and CCC stress by qRT-PCR. The mean expression value was calculated from three independent biological replicates in different samples. The gene expression level was visualized by TBtools; The raw data of relative expression values and standard errors is provided in Table S4.

\section{Discussion}

GA is an important hormone that controls plant growth and development [1-4], thus, its related metabolic and synthetic pathways are of great research interest. As an important member of the 2-oxoglutarate-dependent dioxygenase superfamily, gibberellin oxidase catalyzes the last step of gibberellin metabolism, and its related functions have been verified in many species $[7,16,24,26,28]$. 


\subsection{Gene Identification, Phylogenetic Relationship, Motif Analysis, Gene Structure, and Collinearity Analysis}

In this study, we identified 13 gibberellin oxidase genes belonging to the GA20ox, C19GA20x, and C20GA20x subfamilies. However, we did not detect GA3ox in the L. chinense genome. This shows that $L c G A 30 x$ may have been lost during the evolutionary process. A phylogenetic tree was constructed based on the 72 GAox protein sequences belonging to four subgroups, and the 13 members clustered into three subgroups. Interestingly, AtrGA20ox4 and $O s G A o x 5,7$, and 8 clustered together, indicating that $A \operatorname{trGA20ox4}$ may occupy a special position in evolution based on previous research [36]. The protein sequences of LcGA2ox1,2, and 3 had high homology, suggesting that functional redundancy may exist between the three of them. Most $L c G A o x$ members clustered together with or close to the members of $V v G A o x$ and $A \operatorname{trGAox}$, implying that they are closely related and may have originated from a common ancestor. Mutations of gene function are often related to changes in gene structure and conserved domains [37]. By comparing the gene structure and conserved functional domains between Arabidopsis and L. chinense, we obtained information about the similarities and differences between the two GAox gene families. LcGAox and AtGAox had high similarity in gene structure, indicating that there was a certain degree of conservation between the two families, but the results of the motif analysis showed that some LcGAox (Figure 2) genes had lost or added the domain of 2OG-FeII oxygenase and DIOX_N [27], which may lead to gene functional changes. For example, motifs 12, 2, 5, and 11 were absent in LcGA2ox4, 6, and 8, and the specific motif 10 in C19GA2ox was found in LcGA20ox4. Gene duplication events, as the main factor of genome expansion, mainly include tandem duplication and fragmented duplication [38]. Two fragmented duplications were identified in the LcC19GA2ox gene family, showing that this family experienced an expansion, which may be related to a single whole-genome duplication event in L. chinense. Collinearity analysis with members of the GAox genomic group in grapes, rice, and Arabidopsis showed that there were multiple orthologous genes with L. chinense, and the number of orthologous genes with grape was the largest, indicating a close relationship between the $L c G A o x$ and $V v G A o x$ gene family. The results of the evolutionary selection analysis indicated that the GAox genes in the five species experienced purifying selection.

\subsection{Codon Usage Pattern and cis-Element Analysis}

Through analysis of the GAox codon usage patterns of 21 species, we found that the GAox of angiosperms had obvious codon preference. Among them, the third base of the amino acid encoded by monocotyledons was biased towards $\mathrm{A} / \mathrm{T}$, while that of dicotyledonous plants was biased towards C/G, and this bias was affected by many factors, such as natural selection and mutation pressure. However, LcGAox exhibited no such strong bias. This situation was similar to that of Amborella and Lycopodium, which also showed that $L c G A o x$ was more evolutionarily primitive [39]. By analyzing the cis-elements related to stress and phytohormones in the LcGAox promoter, we found that light-responsive elements were widely present in the promoter region. LcGA2ox1-4, 6, and 7 and LcGA20ox1 and 3 do not directly respond to external abiotic stress, and no GA responsive element was predicted in LcGA2ox5 and LcGA20ox1 and 3, indicating that they are related through other factors.

3.3. The Spatial Expression Pattern of LcGAox and Their Response to Abiotic and Phytohormone Stress Base on the RNA-seq and qRT-PCR

Gibberellin mainly acts on young organs, such as the seeds, young leaves, and apical buds, et al. [11,14]. The transcriptome data of multiple organs indicated that LcGA20ox3, $L c G A 20 x 8$, and LcGA2ox4 were ubiquitously stably expressed in the different organs. LcGA2ox1,2 exhibited stable expression in the developmental process from globular embryo to cotyledon embryo and young leaves. The above results indicated that plants complete the synthesis and degradation of gibberellin by regulating the two genes GA20ox and GA20x, so as to achieve an optimal steady state at a specific organ stage. Interestingly, we 
found the only LcAG20ox3 played an important role in the LcGA20ox gene family, showing that the other $L c G A 200 x$ members either had a specific response or just played an auxiliary role in the synthesis of GA. Combined with the prediction results of the homeostatic regulatory elements and previous studies, it is indicated that gibberellin oxidase has different response modes in response to some stress conditions and hormone treatments. LcGA2ox1 and 4 responded rapidly to PEG and low temperature stress in the roots, stems, and leaves. Under salt stress, the expression of $L c G A 2 o x 1$ in the roots, stems, and leaves peaked within $12 \mathrm{~h}$, while LcGA2ox4 exhibited a lower response in the leaves. Under the stimulation of $A B A, L c G A 1,4$, and 7 had varying responses in the leaves, and LcGA2ox4 specifically responded to $\mathrm{ABA}$ in the stems and upregulated the expression level. The application of exogenous GA activated the expression of $L c G A 2 o x 1,4,7$ in the leaves and $L c G A 2 o x 7$ in the stems, thereby degrading GAs. Under the treatment of a GA inhibitor, the expression of $L c G A 20 x 1,4$ was upregulated in the leaves compared with at $0 \mathrm{~h}$, while the expression of $L c G A 20 x 7$ was downregulated in the stems. These results indicated that $L c G A 20 x 1$ and 4 mainly degraded the GA in the leaves, while the degradation of GA in the stems mainly depended on $L c G A o x 7$, which was indicative of functional specificity.

In summary, our research explored the functional differences of the GAox family in different organs of $L$. chinense, and our results indicate their potential role in the evolution of GAox genes, an aspect worth further study.

\section{Materials and Methods}

\subsection{Identification of Gibberellin Oxidase GA2ox, GA20ox Genes in the L. chinense Genome}

To identify the LcGA2ox, LcGA3ox, and LcGA20ox gene families in L. chinense, the Arabidopsis GA2ox, GA3ox, and GA20ox protein sequences were downloaded from phytozome v12.1 (https:/ / phytozome.jgi.doe.gov/pz/portal.html\#, accessed on 12 June 2021), and the conserved OG-FeII_Oxy (PF03171) and DIOX_N (PF14226) were used as a query. BLASTP and HMMER3.0 software were used to search the target protein sequences in the L. chinense protein database (https: / / hardwoodgenomics.org/Genome-assembly /2630420, accessed on 12 June 2021). The LcGA20x, LcGA2ox, and LcGA3ox protein sequences were further authenticated based on the conserved domains using SMART (http:/ / smart.emblheidelberg. de, accessed on 12 June 2021) and CDD-search (https:/ / www.ncbi.nlm.nih.gov/Structure/ bwrpsb / bwrpsb.cgi, accessed on 12 June 2021). After removing redundant proteins, a total of 213 putative proteins were identified. A phylogenetic tree was constructed using the 213 identified proteins from L. chinense and 16 GAox proteins from Arabidopsis. A total of 13 LcGAox proteins were obtained (Table S1). The gene properties, including the molecular weight $(\mathrm{kDa})$ and isoelectric point $(\mathrm{pI})$ of each protein, were determined using the Compute $\mathrm{pI} / \mathrm{Mw}$ tool on the ExPASy website (Table 1). The subcellular localization of the LcGAox genes was predicted by Cell-PLoc 2.0 (http:/ / www.csbio.sjtu.edu.cn/bioinf/Cell-PLoc-2/, accessed on 12 June 2021).

\subsection{Phylogenetic Tree Construction and the Analysis of Gene Structure, Conserve Motif in Multiple Species and the 3D Structure Determination}

The GAox protein sequences were download from phytozome in Oryza sativa (Os), Amborella trichopoda (Atr), and Vitis vinifera ( $\mathrm{Vv}$ ) based on the AtGAox protein sequences (Tables 1 and S2). The phylogenetic relationships between $L c G A o x$ and the GAox proteins from the other four species were determined using the IQtree software (http:/ / www. iqtree.org/, accessed on 12 June 2021). Sequence relationships were inferred using the maximum likelihood (ML) method, and the optimal model was LG + F + R6 (Figure 1). The $L c G A o x$ sequences were named according to their respective clades. The gene structure information of AtGAox was obtained from the Arabidopsis genomic annotation database in phytozome. The conserved motifs of the $L c G A o x$ and AtGAox proteins were checked using the online multiple expectation maximization for motif elicitation (MEME) program. A hierarchical clustering tree was constructed using the neighbor-joining algorithm with the default parameters of 1000 bootstrap replications in the MEGA 7.0 software [40]. Images 
were illustrated using the TBtools software [41]. The three-dimensional (3D) structure was predicted using the online tool SWISS-MODEL (https://swissmodel.expasy.org/ interactive, accessed on 12 June 2021).

Chromosomal distribution and gene duplication most of the LcGA2ox and LcGA20ox genes were mapped to the $L$. chinense chromosomes based on the location information from the genome database of $L$. chinense by TBtools. The gene repetitive events were analyzed by the Multicollinearity ScanToolkit (MCScanX) [42]. The synteny relationship map of the orthologous GAox genes obtained from L. chinense, Arabidopsis, grape, and rice (Kitaake) were visualized by TBtools. The evolutionary selection relationships of orthologous genes within and between species were analyzed in DnaSP6.

\subsection{Codon Usage Pattern Analysis in GAox Genes}

To systematically analyze the codon usage pattern of GAox genes, we used AtGAox genes as a query in the phytozome database to identify all GAox genes from Physcomitrella patens, Selaginella moellendorffii, Amborella trichopoda, Sorghum bicolor, Oryza sativa, Zea mays, Setaria italica, Arabidopsis thaliana, Brassica oleracea, Zea mays, Medicago truncatula, Gossypium Raimondii, Theobroma cacao, Manihot esculenta, Ricinus communis, Citrus sinensis, Malus domestica, Populus trichocarpa, Cucumis sativus, Solanum lycopersicum, and Vitis vinifera using BLAST (Table S2). The ML method was used to identify different group members by constructing evolutionary trees. The coding sequences of the LcGA2ox and LcGA20ox genes were used to calculate the A, G, C, T, and GC contents at the third site of the synonymous codon (A3s, G3S, C3s, T3s, GC3s content), relative synonymous codon usage (RSCU), and effective numbers of codons (ENC) with the CodonW v.1.4.2 (Table S3). Correlation analysis between codon composition and preference parameters (A3s, T3s, G3s, C3s, GC3s, GC) was carried out using R (www.r-project.org, accessed on 12 June 2021).

\subsection{Analysis of the cis-Acting Element in LcGA2ox and LcGA20ox Gene Families}

The promoter sequences of $L c G A 20 x$ and $L c G A 20 o x$ were extracted from the L. chinense genome database (3000 bp), and the cis-acting elements were predicted and analyzed using the PlantCARE online site [43].

\subsection{Analysis of Gene Expression in Organs by the RNA-seq}

To analyze the expression patterns of the LcGA2ox and LcGA20ox gene families, different organ transcript data for $L$. chinense were downloaded from the NCBI Sequence Read Archive (SRA) with the accession numbers SRR8101040, SRR8101041, SRR8101042, SRR8101043, SRR9945429, SRR9945430, SRR9945433, SRR9948913, SRR9948914, SRR9948915, SRR9948916, SRR9948917, SRR9948918, SRR9948919, SRR9949005, SRR9949006, SRR9949007, SRR9949008, SRR9949009, and SRR9949010. All of the mRNA abundance values were measured by transcripts per million (TPM) based on the L. chinense genomic database [28] and the transcript data (TPM) of $L c G A 2 o x$ and $L c G A 20 o x$ were indicated in a heatmap.

\subsection{Plant Materials Treatment and Expression Analysis by qRT-PCR}

Three-month-old somatic embryo seedlings were cultured in an incubator under white light (16 h light, $8 \mathrm{~h}$ dark) on 3/4MS medium. They were treated separately with $100 \mathrm{mg} / \mathrm{L}$ abscisic acid (ABA), $100 \mathrm{mg} \mathrm{L}^{-1} \mathrm{GA}, 100 \mathrm{mg} \mathrm{L}^{-1}$ chlormequat (CCC), $20 \%$ polyethyleneglycol (PEG)-6000 solution, $100 \mathrm{mM} \mathrm{NaCl}$ solution, or low temperature $\left(4^{\circ} \mathrm{C}\right)$. Mature leaves, stems, and roots were sampled from three biological replicates of the treatment and control plants at $0,6,12,24$, and $48 \mathrm{~h}$ after the trial. Quantitative RT-PCR analysis was used to confirm the expression patterns of LcGA2ox and LcGA20ox in the different organs under the different treatments (Table S4). Total RNA extraction was performed using a KK Fast Plant Total RNA Kit. First-strand cDNA was synthesized from $1.0 \mathrm{mg}$ of RNA with an Evo M-MLV RT Kit with Gdna Clean for qPCRII AG11711 (Accurate Biotechnology (Hunan) Co., Ltd.). The qRT-PCR was carried out using SYBR-green fluorescence in a Roche LightCycler ${ }^{\circledR} 480$ Real-Time PCR System. The $\Delta \Delta C T$ method was used to calculate 
the gene relative expression levels [44]. All qRT-PCR primers were designed by Primer5.0 and are listed in Table S5.

\subsection{Data Analysis}

All data analysis was based on Excel 2019, analysis of variance was based on R, and multiple comparison method was least significant difference (LSD)

\section{Conclusions}

In this study, we identified 13 gibberellin oxidase genes based on publicly-available L. chinense genomic data, which included five GA20ox genes, four C19GA2ox genes, and four C20GA2ox genes. Phylogenetic analysis, gene structure, and conserved motif, and functional site prediction analyses revealed that gibberellin oxidases in L. chinense are conserved and divergent compared with other species. The codon usage pattern indicated that the LcGAox genes had no obvious codon usage bias and were more biased towards primitive species compared with angiosperms. The RNA-seq and qRT-PCR data analysis showed that $L c G A o x$ genes exhibit specific expression patterns in different organs and the stages of somatic embryogenesis. Furthermore, $L c G A 20 x 1$, 4, and 7 can further affect the synthesis or degradation of gibberellin in hybrid Liriodendron by responding to abiotic stress or hormone treatment. In summary, this study provides a basis for further investigating the genetic and functional characteristics of the LcGAox gene family.

Supplementary Materials: The following are available online at https:/ /www.mdpi.com/article/10 $.3390 /$ ijms22137167/s1.

Author Contributions: L.H., J.S., J.C. and T.C. contributed to the management and manuscript review. L.H., Z.H., P.W. and Y.L. designed experiments as well as provided the methodology of data collection and analysis. Z.C. and L.H. helped with the collection of samples. H.Q., L.H. and G.X. performed the experiment. All authors have read and approved the final manuscript.

Funding: This work was supported by the National Natural Science Foundation of China (32071784 and 31770715), State Forestry and grassland administration (2020133106), Priority Academic Program Development of Jiangsu Higher Education Institutions.

Institutional Review Board Statement: Not applicable.

Data Availability Statement: The datasets supporting the conclusions and description of a complete protocol can be found within the manuscript and its additional files. The datasets used and analyzed during the current study are available from the corresponding author on reasonable request.

Conflicts of Interest: The authors declare that they have no competing interests.

\section{References}

1. Webster, A.D. Vigour mechanisms in dwarfing rootstocks for temperate fruit trees. In Proceedings of the 1st International Symposium on Rootstocks for Deciduous Fruit Tree Species, Zaragoza, Spain, 11-14 June 2002; Sanchez, M.A., Webster, A.D., Eds.; International Society for Horticultural Science (ISHS): Leuven, Belgium, 2004; Volumes 1 and 2, pp. $29-41$.

2. Peng, J.R.; Harberd, N.P. The role of GA-mediated signalling in the control of seed germination. Curr. Opin. Plant Biol. 2002, 5, 376-381. [CrossRef]

3. Zhang, N.; Xie, Y.-D.; Guo, H.J.; Zhao, L.-S.; Xiong, H.-C.; Gu, J.-Y.; Li, J.-H.; Kong, F.-Q.; Sui, L.; Zhao, Z.-W.; et al. Gibberellins regulate the stem elongation rate without affecting the mature plant height of a quick development mutant of winter wheat (Triticum aestivum L.). Plant Physiol. Biochem. 2016, 107, 228-236. [CrossRef]

4. Wang, G.-L.; Que, F.; Xu, Z.-S.; Wang, F.; Xiong, A.-S. Exogenous gibberellin altered morphology, anatomic and transcriptional regulatory networks of hormones in carrot root and shoot. Bmc Plant Biol. 2015, 15. [CrossRef]

5. Appleford, N.E.; Lenton, J.R. Gibberellins and leaf expansion in near-isogenic wheat lines containing Rht1 and Rht3 dwarfing alleles. Planta 1991, 183, 229-236. [CrossRef]

6. Giacomelli, L.; Rota-Stabelli, O.; Masuero, D.; Acheampong, A.K.; Moretto, M.; Caputi, L.; Vrhovsek, U.; Moser, C. Gibberellin metabolism in Vitis vinifera L. during bloom and fruit-set: Functional characterization and evolution of grapevine gibberellin oxidases. J. Exp. Bot. 2013, 64, 4403-4419. [CrossRef] [PubMed] 
7. Chen, S.; Wang, X.; Zhang, L.; Lin, S.; Liu, D.; Wang, Q.; Cai, S.; El-Tanbouly, R.; Gan, L.; Wu, H.; et al. Identification and characterization of tomato gibberellin 2-oxidases (GA20xs) and effects of fruit-specific S1GA2ox1 overexpression on fruit and seed growth and development. Hortic. Res. 2016, 3. [CrossRef]

8. Salazar-Cerezo, S.; Martinez-Montiel, N.; Garcia-Sanchez, J.; Perez-y-Terron, R.; Martinez-Contreras, R.D. Gibberellin biosynthesis and metabolism: A convergent route for plants, fungi and bacteria. Microbiol. Res. 2018, 208, 85-98. [CrossRef] [PubMed]

9. Kawai, Y.; Ono, E.; Mizutani, M. Evolution and diversity of the 2-oxoglutarate-dependent dioxygenase superfamily in plants. Plant J. 2014, 78, 328-343. [CrossRef]

10. Sakamoto, T.; Miura, K.; Itoh, H.; Tatsumi, T.; Ueguchi-Tanaka, M.; Ishiyama, K.; Kobayashi, M.; Agrawal, G.K.; Takeda, S.; Abe, K.; et al. An overview of gibberellin metabolism enzyme genes and their related mutants in rice. Plant Physiol. 2004, 134, 1642-1653. [CrossRef] [PubMed]

11. Hedden, P.; Phillips, A.L. Gibberellin metabolism: New insights revealed by the genes. Trends Plant Sci. 2000, 5, 523-530. [CrossRef]

12. Chen, J.; Hao, Z.; Guang, X.; Zhao, C.; Wang, P.; Xue, L.; Zhu, Q.; Yang, L.; Sheng, Y.; Zhou, Y.; et al. Liriodendron genome sheds light on angiosperm phylogeny and species-pair differentiation. Nat. Plants 2019, 5, 18-25. [CrossRef]

13. Eshed, Y.; Lippman, Z.B. Revolutions in agriculture chart a course for targeted breeding of old and new crops. Science 2019, 366, 705. [CrossRef]

14. Hedden, P.; Proebsting, W.M. Genetic analysis of gibberellin biosynthesis. Plant Physiol. 1999, 119, 365-370. [CrossRef]

15. Wang, H.; Jiang, H.; Xu, Y.; Wang, Y.; Zhu, L.; Yu, X.; Kong, F.; Zhou, C.; Han, L. Systematic Analysis of Gibberellin Pathway Components in Medicago truncatula Reveals the Potential Application of Gibberellin in Biomass Improvement. Int. J. Mol. Sci. 2020, 21, 7180. [CrossRef]

16. Do, P.T.; De Tar, J.R.; Lee, H.; Folta, M.K.; Zhang, Z.J. Expression of ZmGA20ox cDNA alters plant morphology and increases biomass production of switchgrass (Panicum virgatum L.). Plant Biotechnol. J. 2016, 14, 1532-1540. [CrossRef]

17. Sasaki, A.; Ashikari, M.; Ueguchi-Tanaka, M.; Itoh, H.; Nishimura, A.; Swapan, D.; Ishiyama, K.; Saito, T.; Kobayashi, M.; Khush, G.S.; et al. Green revolution: A mutant gibberellin-synthesis gene in rice-New insight into the rice variant that helped to avert famine over thirty years ago. Nature 2002, 416, 701-702. [CrossRef]

18. Chiang, H.H.; Hwang, I.; Goodman, H.M. Isolation of the Arabidopsis GA4 locus. Plant Cell 1995, 7, 195-201. [PubMed]

19. Lester, D.R.; Ross, J.J.; Davies, P.J.; Reid, J.B. Mendel's stem length gene (Le) encodes a gibberellin 3 beta-hydroxylase. Plant Cell 1997, 9, 1435-1443. [CrossRef] [PubMed]

20. Israelsson, M.; Mellerowicz, E.; Chono, M.; Gullberg, J.; Moritz, T. Cloning and overproduction of gibberellin 3-oxidase in hybrid aspen trees. Eff. Gibberellin Homeost. Dev. Plant Physiol. 2004, 135, 221-230.

21. Reinecke, D.M.; Wickramarathna, A.D.; Ozga, J.A.; Kurepin, L.V.; Jin, A.L.; Good, A.G.; Pharis, R.P. Gibberellin 3-oxidase Gene Expression Patterns Influence Gibberellin Biosynthesis, Growth, and Development in Pea. Plant Physiol. 2013, 163, 929-945. [CrossRef]

22. Sun, Y.; Zhang, H.; Fan, M.; He, Y.; Guo, P. A mutation in the intron splice acceptor site of a GA3ox gene confers dwarf architecture in watermelon (Citrullus lanatus L.). Sci. Rep. 2020, 10, 14915. [CrossRef]

23. Martinez-Bello, L.; Moritz, T.; Lopez-Diaz, I. Silencing C-19-GA 2-oxidases induces parthenocarpic development and inhibits lateral branching in tomato plants. J. Exp. Bot. 2015, 66, 5897-5910. [CrossRef] [PubMed]

24. Li, R.; Sun, S.; Wang, H.; Wang, K.; Yu, H.; Zhou, Z.; Xin, P.; Chu, J.; Zhao, T.; Wang, H.; et al. FIS1 encodes a GA2-oxidase that regulates fruit firmness in tomato. Nat. Commun. 2020, 11, 1-12. [CrossRef]

25. Schomburg, F.M.; Bizzell, C.M.; Lee, D.J.; Zeevaart, J.A.D.; Amasino, R.M. Overexpression of a novel class of gibberellin 2-oxidases decreases gibberellin levels and creates dwarf plants. Plant Cell 2003, 15, 151-163. [CrossRef]

26. Lv, S.; Yu, D.; Sun, Q.; Jiang, J. Activation of gibberellin 20-oxidase 2 undermines auxin-dependent root and root hair growth in $\mathrm{NaCl}$-stressed Arabidopsis seedlings. Plant Growth Regul. 2018, 84, 225-236. [CrossRef]

27. Rieu, I.; Ruiz-Rivero, O.; Fernandez-Garcia, N.; Griffiths, J.; Powers, S.J.; Gong, F.; Linhartova, T.; Eriksson, S.; Nilsson, O.; Thomas, S.G.; et al. The gibberellin biosynthetic genes AtGA20ox1 and AtGA20ox2 act, partially redundantly, to promote growth and development throughout the Arabidopsis life cycle. Plant J. 2008, 53, 488-504. [CrossRef] [PubMed]

28. Shan, C.; Mei, Z.; Duan, J.; Chen, H.; Feng, H.; Cai, W. OsGA2ox5, a Gibberellin Metabolism Enzyme, Is Involved in Plant Growth, the Root Gravity Response and Salt Stress. PLoS ONE 2014, 9, e87110. [CrossRef]

29. He, H.; Liang, G.; Lu, S.; Wang, P.; Liu, T.; Ma, Z.; Zuo, C.; Sun, X.; Chen, B.; Mao, J. Genome-Wide Identification and Expression Analysis of GA2ox, GA3ox, and GA20ox Are Related to Gibberellin Oxidase Genes in Grape (Vitis vinifera L.). Genes 2019, 10, 680. [CrossRef]

30. Huang, Y.; Wang, X.; Ge, S.; Rao, G.-Y. Divergence and adaptive evolution of the gibberellin oxidase genes in plants. BMC Evol. Biol. 2015, 15, 207. [CrossRef]

31. Roach, P.L.; Clifton, I.J.; Fulop, V.; Harlos, K.; Barton, G.J.; Hajdu, J.; Andersson, I.; Schofield, C.J.; Baldwin, J.E. Crystal structure of isopenicillin $\mathrm{N}$ synthase is the first from a new structural family of enzymes. Nature 1995, 375, 700-704. [CrossRef]

32. Honi, U.; Amin, M.R.; Kabir, S.M.T.; Bashar, K.K.; Moniruzzaman, M.; Jahan, R.; Jahan, S.; Haque, M.S.; Islam, S. Genome-wide identification, characterization and expression profiling of gibberellin metabolism genes in jute. BMC Plant Biol. 2020, 20, 306. [CrossRef] 
33. Angellotti, M.C.; Bhuiyan, S.B.; Chen, G.; Wan, X.-F. CodonO: Codon usage bias analysis within and across genomes. Nucleic Acids Res. 2007, 35, W132-W136. [CrossRef] [PubMed]

34. Chakraborty, S.; Yengkhom, S.; Uddin, A. Analysis of codon usage bias of chloroplast genes in Oryza species: Codon usage of chloroplast genes in Oryza species. Planta 2020, 252, 1-20. [CrossRef] [PubMed]

35. Zhou, J.-H.; Ding, Y.-Z.; He, Y.; Chu, Y.-F.; Zhao, P.; Ma, L.-Y.; Wang, X.-J.; Li, X.-R.; Liu, Y.-S. The Effect of Multiple Evolutionary Selections on Synonymous Codon Usage of Genes in the Mycoplasma bovis Genome. PLoS ONE 2014, 9, e108949. [CrossRef] [PubMed]

36. Han, F.; Zhu, B. Evolutionary analysis of three gibberellin oxidase genesin rice, Arabidopsis, and soybean. Gene 2011, 473, 23-35. [CrossRef] [PubMed]

37. Babenko, V.N.; Rogozin, I.B.; Mekhedov, S.L.; Koonin, E.V. Prevalence of intron gain over intron loss in the evolution of paralogous gene families. Nucleic Acids Res. 2004, 32, 3724-3733. [CrossRef]

38. Leister, D. Tandem and segmental gene duplication and recombination in the evolution of plant disease resistance genes. Trends Genet. 2004, 20, 116-122. [CrossRef]

39. Albert, V.A.; Barbazuk, W.B.; dePamphilis, C.W.; Der, J.P.; Leebens-Mack, J.; Ma, H.; Palmer, J.D.; Rounsley, S.; Sankoff, D.; Schuster, S.C.; et al. The Amborella Genome and the Evolution of Flowering Plants. Science 2013, 342, 1241089.

40. Kumar, S.; Stecher, G.; Tamura, K. MEGA7: Molecular Evolutionary Genetics Analysis Version 7.0 for Bigger Datasets. Mol. Biol. Evol. 2016, 33, 1870-1874. [CrossRef]

41. Chen, C.; Chen, H.; Zhang, Y.; Thomas, H.R.; Frank, M.H.; He, Y.; Xia, R. TBtools: An Integrative Toolkit Developed for Interactive Analyses of Big Biological Data. Mol. Plant 2020, 13, 1194-1202. [CrossRef]

42. Wang, Y.; Tang, H.; DeBarry, J.D.; Tan, X.; Li, J.; Wang, X.; Lee, T.-H.; Jin, H.; Marler, B.; Guo, H.; et al. MCScanX: A toolkit for detection and evolutionary analysis of gene synteny and collinearity. Nucleic Acids Res. 2012, 40, e49. [CrossRef] [PubMed]

43. Lescot, M.; Dehais, P.; Thijs, G.; Marchal, K.; Moreau, Y.; Van de Peer, Y.; Rouze, P.; Rombauts, S. PlantCARE, a database of plant cis-acting regulatory elements and a portal to tools for in silico analysis of promoter sequences. Nucleic Acids Res. 2002, 30, 325-327. [CrossRef] [PubMed]

44. Willems, E.; Leyns, L.; Vandesompele, J. Standardization of real-time PCR gene expression data from independent biological replicates. Anal. Biochem. 2008, 379, 127-129. [CrossRef] [PubMed] 